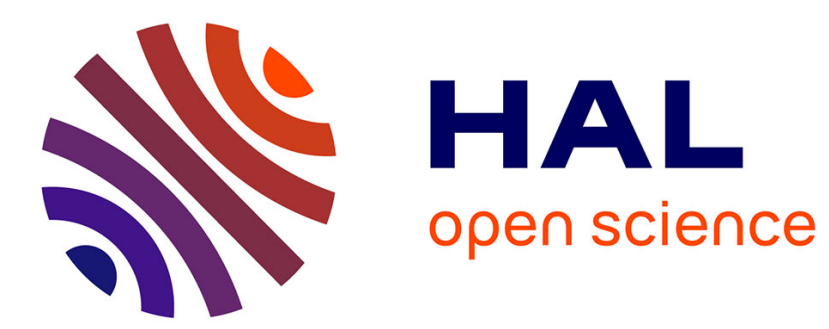

\title{
REGULARITY FOR CRITICAL POINTS OF A NON LOCAL ENERGY \\ Gilles Carbou
}

\section{To cite this version:}

Gilles Carbou. REGULARITY FOR CRITICAL POINTS OF A NON LOCAL ENERGY. Calculus of Variations and Partial Differential Equations, 1997. hal-01728866

\section{HAL Id: hal-01728866 \\ https://hal.science/hal-01728866}

Submitted on 12 Mar 2018

HAL is a multi-disciplinary open access archive for the deposit and dissemination of scientific research documents, whether they are published or not. The documents may come from teaching and research institutions in France or abroad, or from public or private research centers.
L'archive ouverte pluridisciplinaire HAL, est destinée au dépôt et à la diffusion de documents scientifiques de niveau recherche, publiés ou non, émanant des établissements d'enseignement et de recherche français ou étrangers, des laboratoires publics ou privés. 


\title{
REGULARITY FOR CRITICAL POINTS OF A NON LOCAL ENERGY
}

\section{GILLES CARBOU}

\author{
Centre de Mathématiques et \\ de Leurs Applications (URA 1611) \\ Ecole Normale Supérieure de Cachan \\ 61, avenue du Président Wilson \\ 94235 CACHAN Cedex
}

France

\begin{abstract}
: we study the regularity of critical points of an energy which stems from micromagnetism theory. First we show that in dimension two critical points are smooth in $B^{2}$. In the three dimensional case we prove that the stationary critical points of the energy are smooth except in a subset of one dimensional Hausdorff measure zero. The particularity of this work is the non local character of one term of the energy.
\end{abstract}

\section{Introduction.}

\subsection{Micromagnetism theory.}

In the micromagnetism theory, presented by J. Miltat in [19] and by W. F. Brown in [6], a soft magnetic material is characterized by a spontaneous magnetization defined by a magnetic moment per unit volume denoted $M(x)$. This magnetic moment links the magnetic field $H$ and the magnetic induction $B$ by the relation

$$
B=H+4 \pi M .
$$

Furthermore the norm of $M$ is constant and we write $M(x)=M_{s} u(x)$ where $|u(x)|=1$.

The energy associated with a configuration $M$ consists of the sum of five terms.

$$
E_{\text {total }}(M)=E_{\text {exch }}+E_{H_{a}}+E_{H_{d}}+E_{K}+E_{M E} .
$$

The first one is called the exchange energy. It comes from Heisenberg's model of the interaction energy between two spins :

$$
E_{\text {exch }}=\frac{1}{2} \int|\nabla M|^{2}
$$

The two following terms are deduced from Maxwell's equations :

$$
\operatorname{curl} H=j \quad \operatorname{div} B=0 .
$$


We decompose $H$ into two terms : $H=H_{a}+H_{d}$. The applied field $H_{a}$ arises from external current distributions $j$ :

$$
\operatorname{curl} H_{a}=j \quad \operatorname{div} H_{a}=0 .
$$

The demagnetizing field satisfies :

$$
\operatorname{curl} H_{d}=0 \quad \operatorname{div}\left(H_{d}+4 \pi M\right)=0 .
$$

The energy induced by $H_{a}$ and $H_{d}$ are

$$
E_{H_{a}}=-\int H_{a} M \quad E_{H_{d}}=-\frac{1}{2} \int H_{d} M
$$

The last two terms pattern the relations between the magnetic properties and crystal orientations. The anisotropy energy $E_{K}$ reflects the existence of preferential axes of magnetization. The magnetoelastic energy $E_{M E}$ expresses the link between elastic strains and the direction of $M$.

\subsection{Statement of the results.}

In this article we study critical points of an energy defined for $u \in H^{1}\left(B^{3}, S^{2}\right)$ by

$$
E(u)=\frac{1}{2} \int_{B^{3}}|\nabla u|^{2}-\frac{1}{2} \int_{B^{3}} H \cdot u+\int_{B^{3}} F(u)
$$

where $H \in L^{2}\left(\mathbb{R}^{3}, \mathbb{R}^{3}\right)$ is the solution of the following non local problem:

$$
\diamond\left\{\begin{array}{l}
\operatorname{curl} H=0 \\
\operatorname{div}(H+\bar{u})=0 \text { in } \mathcal{D}^{\prime}\left(\mathbb{R}^{3}\right)
\end{array}\right.
$$

where $\bar{u}$ is equal to $\mathrm{u}$ in $B^{3}$ and is zero out of $B^{3}$.

We assume that $F \in \mathcal{C}^{1}\left(\mathbb{R}^{3}, \mathbb{R}\right)$ and satisfies

$$
\forall \xi \in \mathbb{R}^{3},|F(u)| \leq K\left[1+|\xi|^{6}\right] .
$$

We denote

$$
\nabla F(\xi)=f(\xi)
$$

REMARK 1 : the energy $E$ is obtained from the physical model taking all the physical constants equal to 1 . So the first term of $E$ represents the exchange energy, the non local term corresponds to the demagnetizing energy, and the local term contains applied, anisotropy and magnetoelastic energies.

REMARK 2 : we will prove that the non local term of $E$ satisfies :

$$
-\int_{B^{3}} H \cdot u=\int_{\mathbb{R}^{3}}|H|^{2} .
$$

Hence with the hypothesis about $F$, the proof of the existence of minimizers of the energy is obvious. 
Critical points of $E$ satisfy the Euler equation :

$$
-\triangle u-u\|\nabla u\|^{2}-H+<u, H>u+f(u)-<u, f(u)>u=0 .
$$

The first term of $E$ is the Dirichlet energy, i.e. the energy which the critical points are the harmonic maps with values in a riemannian manifold. In the next subsection we will recall the regularity results concerning harmonic maps. We obtain in this article the same results concerning now the critical points of $E$.

Our first result is a regularity theorem in dimension two.

THEOREM 1. Let $u \in H^{1}\left(B^{2}, S^{2}\right)$ be a critical point of $E$. Then $u$ is smooth on $B^{2}$.

REMARK 3 : if we suppose that the domain is a cylinder $B^{2} \times \mathbb{R}$, we can consider a magnetic moment per unit volume invariant by translations parallel to the $z$-axis (see [19]). In this case we work with maps defined on a two dimensional domain.

The regularity theory for critical points of quadratic functionals in dimension two has considerably progressed since the theorems of Morrey in 1948 (see [20]). One of the most important results is proved in [15] by F. Hélein. It concerns the regularity of harmonic maps defined in an open set of $\mathbb{R}^{2}$ and with values in a Riemannian manifold. One can find a generalization of this result which concerns harmonic sections in [7]. Besides F. Bethuel shows in [3] a result of regularity for the solutions of prescribed mean curvature surfaces equation. For the interior regularity we use mainly here the works of F. Hélein about the harmonic maps into the sphere (see [16]).

Our second result is the following

THEOREM 2. Assume $u \in H^{1}\left(B^{3}, S^{2}\right)$ is a stationary critical point of $E$. Then there exists an open subset $V \subset B^{3}$ such that $u$ is smooth in $V$ and $\mathcal{H}^{1}\left(B^{3}-V\right)=0$, where $\mathcal{H}^{1}$ denotes one dimensional Hausdorff measure.

Note that a stationary critical point of $E$ is a critical point of $E$ when we allow variations both acting on the target manifold (as in the end of the section) and acting on the source manifold. A precise definition will be given in section 3 .

Theorem two is similar to the result of Evans concerning stationary harmonic maps with values in a sphere (see [10]

We recall now the regularity results concerning weakly harmonic maps with values in a riemannian manifold.

\subsection{Harmonic maps.}

Let $\mathcal{M}$ be a compact submanifold of $\mathbb{R}^{n}$. We consider the Dirichlet energy

$$
F(u)=\int_{\Omega}\|\nabla u\|^{2}
$$

defined for $u \in H^{1}(\Omega, \mathcal{M})$ where $\Omega$ is an open set of $\mathbb{R}^{n}$. 
DEFINITION : the critical points of $F$ are called harmonic maps with values in $\mathcal{M}$. They satisfy the equation :

$$
-\triangle u-A_{u}(\nabla u, \nabla u)=0 \text { in } \mathcal{D}^{\prime}(\Omega)
$$

where $A_{u}$ is the second fundamental form of the embedding of $\mathcal{M}$ in $\mathbb{R}^{n}$.

For example when $\mathcal{M}=S^{n-1}$, harmonic maps with values in $S^{n-1}$ satisfy

$$
-\triangle u-u\|\nabla u\|^{2}=0 \text {. }
$$

One of the most interesting topic concerning harmonic maps is the problem of their regularity. We recall that the standard elliptic estimates give an improvement of regularity when the laplacian of $u$ is in $L^{p}$ with $1<p<+\infty$. In the case of harmonic maps these hypothesis are not satisfied since we obtain for example when $\mathcal{M}$ is the unit sphere

$$
-\triangle u=u\|\nabla u\|^{2} \in L^{1} .
$$

In fact the regularity of harmonic maps depends on the dimension of the domain $\Omega$.

When $\Omega$ is two-dimensional harmonic maps defined on $\Omega$ with values in a compact riemannian manifold are regular. It is a theorem proved by F. Hélein in [15].

When the dimension of the source is greater than three, harmonic maps are not regular. A counter-example is given by $\frac{x}{|x|}$ which is harmonic with values in the sphere.

The first results of partial regularity in dimension greater than two for the minima of quadratic functionals have been obtained in the case of minimizing harmonic maps by $\mathrm{R}$. Schoen and K. Uhlenbeck (see [23]) and by Hardt, Kinderlerher, Lin (see [14]). Furthermore Evans proves in [10] a result of partial regularity when $u$ is only a stationary harmonic map with values in $S^{n-1}$. This result is generalized by Bethuel in [4]. Moreover T. Rivière builds in [22] harmonic maps totally discontinuous.

\subsection{Outline of the paper.}

The paper is organized as follows :

In the end of this part we compute the Euler equations of the problem.

Theorem 1 will be proved in the second part. We shall use compensation results proved by Wente and which enable the demonstration of the regularity in dimension two of harmonic maps with values in a "very symmetric" submanifold (see Hélein's article [17]).

The third part is devoted to the proof of the partial regularity for the stationary critical points of $E$ in the three-dimensional case.

We follow in this part the ideas of L.C. Evans (see [10]). We first find a monotonicity inequality. Afterwards we show a theorem of $\varepsilon$-regularity: if $\tilde{E}\left(x_{0}, r\right)<\varepsilon_{0}$ for $r$ little enough then $u$ is smooth in a neighbourhood of $x_{0}$, where

$$
\tilde{E}\left(x_{0}, r\right)=\frac{1}{r} \int_{B\left(x_{0}, r\right)}|\nabla u|^{2} .
$$


In order to prove this theorem one needs a result of compactness in the strong $H^{1}$ topology which uses the $\mathcal{H}^{1}-B M O$ duality. As it is remarked in Evans' article (see [10]) these spaces play a very important role in the regularity problems. Lastly we conclude the proof with a classical covering argument.

In the fourth part we give without proof further results about the minimizers of $E$.

Compared with the theorems relating to weakly harmonic maps, the second term of the energy entails several difficulties. The first one is the non local character of this term which keeps us from getting easily a monotonicity formula. The second difficulty is that the homogeneity of the third term in the Euler equation is different from the homogeneity of the two first terms. This fact carries away new technical difficulties for the proof of the $\varepsilon$-regularity. Last but not least, because of the term $H$ the calculation of the equation satisfied by stationary critical points of $E$ is quite delicate since we have to write $H$ in the shape of a convolution of div $u$ with a very singular kernel.

REMARK 4 : in a recent paper (see [13]) R. Hardt and D. Kinderlehrer establish the partial regularity of the minima and study their singularities. The point of view and the proofs of their article are very interesting and very different from the content of my work.

\section{NOTATIONS :}

$* B^{N}$ is the unit ball of $\mathbb{R}^{N}$.

$* S^{p}$ is the unit sphere of $\mathbb{R}^{p+1}$.

$* B(x, r)$ is the ball centered in $x$ of radius $r$.

* If $\Omega$ is an open set of $\mathbb{R}^{N}$ and $\mathcal{M}$ is a submanifold of $\mathbb{R}^{n}$ we write

$$
H^{1}(\Omega, \mathcal{M})=\left\{u \in H^{1}\left(\Omega, \mathbb{R}^{n}\right) \text { such that } u(x) \in \mathcal{M} \text { a.e. }\right\} .
$$

* If $\Omega$ is an open set of $\mathbb{R}^{N}, \mathcal{D}\left(\Omega, \mathbb{R}^{N}\right)$ is the space of the smooth function whose support is a compact subset of $\Omega$ with values in $\mathbb{R}^{N}$. We denote $\mathcal{D}^{\prime}(\Omega)$ the space of the distributions on $\Omega$.

\subsection{Euler equations.}

\subsubsection{Study of $H$.}

Let us first make two remarks about the map $u \mapsto H$.

LEMMA 1. Let $u \in H^{1}\left(B^{3}, S^{2}\right)$. Let $H \in L^{2}\left(\mathbb{R}^{3}, \mathbb{R}^{3}\right)$ be the solution of $\diamond$. Then

$$
H \in \bigcap_{1 \leq p<\infty} L^{p}\left(\mathbb{R}^{3}\right)
$$

Proof : we note that $u \in L^{\infty}\left(\mathbb{R}^{3}\right)$ hence $\operatorname{div} u \in W^{-1, \infty}$. We consider $\Phi \in H^{1}\left(\mathbb{R}^{3}, \mathbb{R}^{3}\right)$ such that $\triangle \Phi=-\operatorname{div} u$ in $\mathbb{R}^{3}$. We have that $\Phi \in \bigcap_{1 \leq p<\infty} W^{1, p}$. Therefore since $H=\nabla \Phi$ we obtain

$$
H \in \bigcap_{1 \leq p<\infty} L^{p}\left(\mathbb{R}^{3}\right) .
$$


Furthermore we remark that for all $p \in(1,+\infty)$ there exists a constant $K_{p}$ such that

$$
\|H\|_{L^{p}} \leq K_{p}\|u\|_{L^{p}}
$$

LEMMA 2. The map $u \mapsto H$ where $H$ is the solution of $\diamond$ is self-adjoint for the $L^{2}$-scalar product.

Proof : let $u$ and $v$ in $H^{1}\left(B^{3}, S^{2}\right)$. We consider $\Phi($ resp. $\Psi)$ in $H^{1}\left(\mathbb{R}^{3}, \mathbb{R}^{3}\right)$ such that $\triangle \Phi=-\operatorname{div} u$ in $\mathbb{R}^{3}$ (resp. $\triangle \Psi=-\operatorname{div} v$ in $\mathbb{R}^{3}$ ). We set $H=\nabla \Phi$ and $L=\nabla \Psi$. We have

$$
\begin{gathered}
\int_{B^{3}} v \cdot H=\int_{\mathbb{R}^{3}} v \cdot \nabla \Phi \\
=-\int_{\mathbb{R}^{3}} \operatorname{div} v \cdot \Phi=-\int_{\mathbb{R}^{3}} \triangle \Psi . \Phi \\
=\int_{\mathbb{R}^{3}} \nabla \Psi . \nabla \Phi .
\end{gathered}
$$

Hence we obtain that

$$
\int_{B^{3}} v \cdot H=\int_{B^{3}} L \cdot u
$$

i.e. $u \mapsto H$ is self-adjoint in $L^{2}$.

\subsubsection{Euler equations.}

We are going now to compute the Euler equation satisfied by the critical points of $E$. Let $\varphi$ be an element of $\mathcal{D}\left(B^{3}, \mathbb{R}^{3}\right)$. We set

$$
u_{t}=\frac{u+t \varphi}{|u+t \varphi|}=u+t(\varphi-<u, \varphi>u)+\mathcal{O}\left(t^{2}\right)
$$

and we note

$$
\delta u=\varphi-<u, \varphi>u .
$$

Let $H_{t}$ be the solution of $\diamond$ associated with $u_{t}$. We set now

$$
E\left(u_{t}\right)=\frac{1}{2} \int_{B^{3}}\left|\nabla u_{t}\right|^{2}-\frac{1}{2} \int_{B^{3}} H_{t} \cdot u_{t}+\int_{B^{3}} F\left(u_{t}\right) .
$$

We remark that

$$
H_{t}=H+t \delta H+\mathcal{O}\left(t^{2}\right)
$$

where $\delta H$ satisfies

$$
\left\{\begin{array}{l}
\operatorname{curl} \delta H=0 \\
\operatorname{div}(\delta H+\overline{\delta u})=0 \text { in } \mathcal{D}^{\prime}\left(\mathbb{R}^{3}\right)
\end{array}\right.
$$

Hence with these notations we obtain

$$
E\left(u_{t}\right)=E+t \delta E+\mathcal{O}\left(t^{2}\right)
$$


where

$$
\delta E=\int_{B^{3}} \nabla u \nabla \delta u-\frac{1}{2} \int_{B^{3}} \delta H . u-\frac{1}{2} \int_{B^{3}} \delta u \cdot H+\int_{B^{3}} f(u) . \delta u .
$$

* Computation of the first term :

$$
\begin{gathered}
\int_{B^{3}} \nabla u \nabla \delta u=\int_{B^{3}} \nabla u \nabla(\varphi-<u, \varphi>u) \\
=\int_{B^{3}} \nabla u \nabla \varphi-\int_{B^{3}} \nabla u \cdot \nabla u \cdot\left\langle u, \varphi>-\int_{B^{3}} \nabla u \cdot u \nabla<u, \varphi>\right. \\
=\int_{B^{3}} \nabla u \nabla \varphi-\nabla u . \nabla u \cdot<u, \varphi>
\end{gathered}
$$

since $u$ is orthogonal to $\nabla u$. Hence

$$
\int_{B^{3}} \nabla u \nabla \delta u=\mathcal{D}^{\prime}<-\triangle u-u|\nabla u|^{2}, \varphi>_{\mathcal{D}} .
$$

* Computation of the second term :

From the second lemma we know that $u \mapsto H$ is self-adjoint hence

$$
-\frac{1}{2} \int_{B^{3}} \delta H \cdot u=-\frac{1}{2} \int_{B^{3}} \delta u \cdot H=-\frac{1}{2} \int_{B^{3}}<\varphi, H-\langle u, H>u>.
$$

The computation of the last two terms is obvious.

Therefore the critical points of $E$ satisfy the following Euler equation

$$
-\triangle u-u\|\nabla u\|^{2}-H+<u, H>u+f(u)-<u, f(u)>u=0 \text { in } \mathcal{D}^{\prime}\left(B^{3}\right) \text {. }
$$

\section{Proof of theorem 1.}

\subsection{Compensation result.}

We shall use in this part a result of Wente. It is proved in detail in [5] :

THEOREM 3. Let $\Omega$ be an open set of $\mathbb{R}^{2}$. Let $u \in H^{1}(\Omega)$ such that

$$
\triangle u=a_{x} b_{y}-a_{y} b_{x}
$$

where $a$ and $b$ are in $H^{1}(\Omega)$. Then $u$ is continuous in $B^{2}$.

REMARK 5 : using this result the proof of the regularity of harmonic maps in dimension two with values into $S^{n}$ is quasi immediate.

REMARK 6 : with these hypothesis the result of Wente gives us an estimate of the form :

$$
\|u\|_{L^{\infty}(\Omega)} \leq C(\Omega)\|\nabla a\|_{L^{2}(\Omega)} \cdot\|\nabla b\|_{L^{2}(\Omega)}
$$

where $C(\Omega)$ is a constant which only depends on the conformal class of the open set $\Omega$. Sami Baraket studies in [2] the best constant in this inequality. 


\subsection{Proof of theorem 1.}

We shall write the Euler equation on the form of a conservation law in order to put in evidence curls and to be able to apply Wente's theorem.

Since $\sum_{j} \nabla u^{j} \cdot u^{j}=0$, we can write the Euler equations verified by $u$ on the form

$$
-\triangle u^{i}=\nabla u^{j}\left(u^{i} \nabla u^{j}-u^{j} \nabla u^{i}\right)+\psi_{i}
$$

where

$$
\psi_{i}=H^{i}-u^{i}<H, u>-f^{i}(u)+u^{i}<f(u), u>.
$$

We know that $u$ is uniformly bounded by 1 . Furthermore

$$
H \in L^{2}\left(B^{2}\right) \text { and } f(u) \in L^{\infty} .
$$

We remark then that

$$
\operatorname{div}\left(u^{i} \nabla u^{j}-u^{j} \nabla u^{i}\right)=-u^{i} \psi^{j}+u^{j} \psi^{i} \in L^{2}\left(B^{2}\right) .
$$

Let $b^{i j} \in W^{1,2}\left(B^{2}\right)$ be a solution of

$$
\operatorname{div} b^{i j}=-u^{i} \psi^{j}+u^{j} \psi^{i} .
$$

In particular $b^{i j} \in L^{6}\left(B^{2}\right)$.

Since

$$
\operatorname{div}\left(u^{i} \nabla u^{j}-u^{j} \nabla u^{i}-b^{i j}\right)=0
$$

there exist $c_{i j} \in H^{1}\left(B^{2}\right)$ such that

$$
u^{i} \nabla u^{j}-u^{j} \nabla u^{i}-b^{i j}=\operatorname{curl} c_{i j} .
$$

Hence

$$
-\triangle u^{i}=\nabla u^{j} . \operatorname{curl} c_{i j}-\nabla u^{j} \cdot b^{i j}+\psi^{i}
$$

We decompose $u^{i}$ in two parts : $u^{i}=\alpha_{1}^{i}+\alpha_{2}^{i}$ where

$$
\begin{gathered}
-\triangle \alpha_{1}^{i}=\nabla u^{j} \cdot \operatorname{curl} c_{i j} \\
-\triangle \alpha_{2}^{i}=-\nabla u^{j} \cdot b^{i j}+\psi^{i} \in L^{\frac{3}{2}} \text { with } \alpha_{2}^{i}=0 \text { on } \partial B^{2} .
\end{gathered}
$$

Using theorem 3 we obtain that $\alpha_{1}^{i}$ is continuous. Furthermore we know from standard elliptic estimates that $\alpha_{2}^{i}$ is in $W^{2, \frac{3}{2}}\left(B^{2}\right)$ and from the Sobolev embedding that $\alpha_{2}^{i} \in \mathcal{C}^{0, \alpha}$.

So $u$ is continuous and we conclude the proof by a result of Ladyzenskaya and Ural'ceva (see [18]).

\section{Proof of theorem 3.}

We recall that we are in the case $u \in H^{1}\left(B^{3}, S^{2}\right)$. 


\subsection{Stationary critical points of $E$.}

Roughly speaking a stationary critical point of $E$ is a critical point of $E$ when we allow variations both acting on the target manifold and also on the source manifold :

DEFINITION : a map $u \in H^{1}\left(B^{3}, S^{2}\right)$ is a stationary critical point of $E$ if $u$ is a critical point of $E$ which satisfies the following property. If $\chi_{t}$ is a smooth one-parameter family of diffeomorphisms of $B^{3}$ satisfying $\chi_{0}=I d_{B^{3}}$ and $\chi_{t \mid \partial B^{3}}=I d_{\mid \partial B^{3}}$ then

$$
\frac{d}{d t} E\left(u \circ \chi_{t}\right)_{\mid t=0}=0
$$

Let $u$ be a stationary critical point of $E$. We shall compute the equations verified by $u$.

Let $\xi$ be a smooth vectors field with compact support in $B^{3}$. We set

$$
u_{t}(x)=u(x+t \xi(x))
$$

where $t$ is little enough. We consider $\Phi_{t} \in H^{1}\left(\mathbb{R}^{3}\right)$ the solution of

$$
\triangle \Phi_{t}=-\operatorname{div} \overline{u_{t}}
$$

when $\bar{u}_{t}=u_{t}$ in $B^{3}$ and zero out of $B^{3}$. We remark that for all $t$, the map $\Phi_{t}$ is in $W^{1, p}\left(\mathbb{R}^{3}\right)$ for $1<p<\infty$ and from the Sobolev embedding $\Phi_{t} \in L^{\infty}\left(\mathbb{R}^{3}\right)$.

We set $H_{t}=\nabla \Phi_{t}$ and we have

$$
E\left(u_{t}\right)=\frac{1}{2} \int_{B^{3}}\left\|\nabla u_{t}\right\|^{2}-\frac{1}{2} \int_{B^{3}} H_{t} \cdot u_{t}+\int_{B^{3}} F\left(u_{t}\right) .
$$

Let us compute $\frac{d}{d t} E\left(u_{t}\right)_{\mid t=0}$.

First part of the energy.

The computation of $A=\frac{d}{d t}\left(\frac{1}{2} \int_{B^{3}}\left\|\nabla u_{t}\right\|^{2}\right)_{\mid t=0}$ is classical (it concerns stationary harmonic maps). One obtains :

$$
A=-\frac{1}{2} \int_{B^{3}}\left|\nabla u_{t}\right|^{2} \operatorname{div} \xi+\int_{B^{3}} \sum_{i, \alpha, \beta} u_{x_{\alpha}}^{i} u_{x_{\beta}}^{i} \xi_{x_{\alpha}}^{\beta} .
$$

\section{Second term of the energy.}

Let us compute $\frac{d}{d t}\left(-\frac{1}{2} \int_{\mathbb{R}^{3}} u_{t} \cdot H_{t}\right)_{\mid t=0}=0$.

We consider $G(r)$ the Laplace kernel in $\mathbb{R}^{3}$ :

$$
G(r)=\frac{C}{r}
$$

Let $\phi \in H^{1}\left(\mathbb{R}^{3}, \mathbb{R}\right)$ be the solution of

$$
\triangle \phi=\operatorname{div} \bar{v} \text { in } \mathcal{D}^{\prime}\left(\mathbb{R}^{3}\right)
$$


where $\bar{v}$ is equal to $v$ in $B^{3}$ and is zero out of this ball. We have

$$
\phi(x)=\int_{B^{3}} G(|x-y|) \operatorname{div} v(y) d y-\int_{\partial B^{3}} G(|x-y|)<v(y), n(y)>d \sigma(y) .
$$

This expression is right in the 3 -dimensional case since the laplace kernel in $\mathbb{R}^{3}$ is in $L^{2}\left(B^{3}\right)$, hence the previous integral exists.

We note that

$$
\operatorname{div} u_{t}(x)=\operatorname{div} u(x+t \xi(x))+t \sum_{i, j=1}^{3} u_{x_{j}}^{i}(x+t \xi(x)) \cdot \xi_{x_{i}}^{j}(x) .
$$

We obtain

$$
B_{t}=-\frac{1}{2} \int_{B^{3}} u_{t} \cdot H_{t}=\frac{1}{2} \int_{B^{3}} \operatorname{div} u_{t} \cdot \Phi_{t}-\frac{1}{2} \int_{\partial B^{3}}\left(u_{t} \cdot n\right) \Phi_{t} d \sigma,
$$

now we remark that $u_{t}(x)=u(x)$ on $\partial B^{3}$ hence with the previous integral formulation of $\Phi_{t}$ we deduce the formula

$$
\begin{gathered}
B_{t}=\frac{1}{2} \iint_{x, y \in B^{3}} G(|x-y|)\left(\operatorname{div} u(.+t \xi)+t \sum_{i, j=1}^{3} u_{x_{j}}^{i}(.+t \xi) \xi_{x_{i}}^{j}\right)(x) \\
\cdot\left(\operatorname{div} u(.+t \xi)+t \sum_{i, j=1}^{3} u_{x_{j}}^{i}(.+t \xi) \xi_{x_{i}}^{j}\right)(y) d x d y \\
-\frac{1}{2} \int_{x \in B^{3}} \int_{y \in \partial B^{3}}\left(\operatorname{div} u(.+t \xi)+t \sum_{i, j=1}^{3} u_{x_{j}}^{i}(.+t \xi) \cdot \xi_{x_{i}}^{j}\right)(x) G(|x-y|)(u(y), n(y)) d x d \sigma(y) \\
-\frac{1}{2} \int_{x \in \partial B^{3}} \int_{y \in B^{3}}\left(\operatorname{div} u(.+t \xi)+t \sum_{i, j=1}^{3} u_{x_{j}}^{i}(.+t \xi) \cdot \xi_{x_{i}}^{j}\right)(y) G(|x-y|)(u(x), n(x)) d \sigma(x) d y \\
+\frac{1}{2} \int_{x \in \partial B^{3}} \int_{y \in \partial B^{3}} G(|x-y|)(u(x), n(x))(u(y), n(y)) d \sigma(x) d \sigma(y) .
\end{gathered}
$$

We note that we have used the Fubini theorem which is valid in this case since the map $G(|.-y|)$ is in $L^{2}\left(B^{3}\right)$ for all $y$ in $B^{3}$.

We now do a change of chart in the integrals on $B^{3}$ setting

$$
X=x+t \xi(x) \quad(\text { and } Y=y+t \xi(y)) .
$$

The jacobian of this change of chart in $x$ is equal to $1+t \operatorname{div} \xi(x)+\mathcal{O}\left(t^{2}\right)$ (and the same for $y$ ). 
After a somewhat long computation we get

$$
\begin{gathered}
{\frac{d B_{t}}{d t}}_{\mid t=0}=-\int_{B^{3}} \operatorname{div} \xi \cdot \operatorname{div} u . \Phi+\int_{B^{3}} \Phi \sum_{i, j} u_{x_{j}}^{i} \xi_{x_{i}}^{j} \\
+\frac{1}{2} \int_{x, y \in B^{3}} \operatorname{div} u(x) \operatorname{div} u(y) G^{\prime}(|x-y|)\left(\frac{x-y}{|x-y|}, \xi(x)-\xi(y)\right) d x d y .
\end{gathered}
$$

We need to study the last term of this expression :

$$
C=\frac{1}{2} \int_{x, y \in B^{3}} \operatorname{div} u(x) \operatorname{div} u(y) G^{\prime}(|x-y|)\left(\frac{x-y}{|x-y|}, \xi(x)-\xi(y)\right) d x d y .
$$

We note that this integral exists because $\xi$ is smooth and so $\frac{\xi(x)-\xi(y)}{|x-y|}$ is uniformly bounded in $B^{3} \times B^{3}$. In return we can not split $C$ because we are not sure that the integral

$$
\frac{1}{2} \int_{x, y \in B^{3}} \operatorname{div} u(x) \operatorname{div} u(y) G^{\prime}(|x-y|)\left(\frac{x-y}{|x-y|}, \xi(x)\right) d x d y
$$

exists. Hence we will use singular integrals theory (Cf [24]).

We write for $\varepsilon>0$

$$
M_{\varepsilon}=B^{3} \times B^{3} \backslash\{(a, b) \text { s. t. }\|a-b\| \leq \varepsilon\}
$$

and we have

$$
C=\frac{1}{2} \lim _{\varepsilon \rightarrow 0}\left(\int_{M_{\varepsilon}} \operatorname{div} u(x) \operatorname{div} u(y) G^{\prime}(|x-y|)\left(\frac{x-y}{|x-y|}, \xi(x)-\xi(y)\right) d x d y\right) .
$$

Now we can cut this integral in two terms and we obtain

$$
C=\lim _{\varepsilon \rightarrow 0}\left(\int_{M_{\varepsilon}} \xi(x) \operatorname{div} u(x) \frac{x-y}{|x-y|} G^{\prime}(|x-y|) \operatorname{div} u(y) d y d x\right)
$$

i.e.

$$
C=\lim _{\varepsilon \rightarrow 0}\left[\int_{x \in B^{3}} \xi(x) \operatorname{div} u(x)\left(\int_{y \in B^{3} \backslash B(x, \varepsilon)} \frac{x-y}{|x-y|} G^{\prime}(|x-y|) \operatorname{div} u(y) d y\right) d x\right] .
$$

We set now

$$
L_{\varepsilon}(x)=\int_{y \in B^{3} \backslash B(x, \varepsilon)} \frac{x-y}{|x-y|} G^{\prime}(|x-y|) \operatorname{div} u(y) d y,
$$

we set $L(x)=\lim _{\varepsilon \rightarrow 0} L_{\varepsilon}(x)$ and we know from a result of Stein (see [24]) that $L_{\varepsilon}$ converges to $L$ in $L^{2}\left(B^{3}\right)$.

Furthermore we know that $x \mapsto \xi(x) \operatorname{div} u(x)$ is in $L^{2}\left(B^{3}\right)$ so we get that

$$
C=\int_{B^{3}} \xi(x) \operatorname{div} u(x) L(x) d x .
$$




\section{Last term of $E$.}

$$
\frac{d}{d t}\left(\int_{B^{3}} F\left(u_{t}\right)\right)=\int_{B^{3}} f(u(x+t \xi(x)) \cdot[\nabla u(x+t \xi(x)) \cdot \xi(x)] .
$$

We do the change of chart $X=x+t \xi(x)$ and we obtain that

$$
\frac{d}{d t}\left(\int_{B^{3}} F\left(u_{t}\right)\right)_{\mid t=0}=\int_{B^{3}} f(u(x)) \cdot[\nabla u(x) \cdot \xi(x)] .
$$

As a conclusion the stationary critical points of $E$ satisfy

$$
\begin{gathered}
-\frac{1}{2} \int_{B^{3}}\left|\nabla_{t} u\right|^{2} \operatorname{div} \xi+\int_{B^{3}} \sum_{i, \alpha, \beta} u_{x_{\alpha}}^{i} u_{x_{\beta}}^{i} \xi_{x_{\alpha}}^{\beta}-\int_{B^{3}} \operatorname{div} \xi \cdot \operatorname{div} u \cdot \Phi \\
+\int_{B^{3}} \Phi \sum_{i, j} u_{x_{j}}^{i} \xi_{x_{i}}^{j}+\int_{B^{3}} \xi(x) \operatorname{div} u(x) L(x) d x+\int_{B^{3}} f(u(x)) \cdot[\nabla u(x) \cdot \xi(x)]=0
\end{gathered}
$$

for all $\xi \in \mathcal{D}\left(B^{3}, \mathbb{R}^{3}\right)$.

Let us study $L$ with more precision. We are going to show that $L$ belongs to all the $L^{p}$ spaces for $1<p<\infty$. We have

$$
L(x)=-H(x)+\int_{\partial B^{3}} \frac{x-y}{|x-y|} G^{\prime}(|x-y|)(u(y), n(y)) d \sigma(y)=-H(x)+v(x) .
$$

We already know that $H$ is in all the $L^{p}$ spaces for $1<p<\infty$. We have now

$$
\forall x \in B^{3},|v(x)| \leq K \int_{\partial B^{3}} \frac{d \sigma(y)}{|x-y|^{2}} \leq K \log (1-\|x\|)
$$

and we know that this last map is in all the $L^{p}$ spaces for $1<p<\infty$ on $B^{3}$.

\subsection{Monotonicity formula.}

Let $\mathrm{u}$ be a critical stationary point of $E$. We shall prove that $u$ satisfies a monotonicity formula similar to the inequality satisfied by stationary harmonic maps (see [10]).

We set

$$
\tilde{E}(x, r)=\frac{1}{r} \int_{B(x, r)}|\nabla u|^{2} .
$$

PROPOSITION 1. There exists a constant $\Gamma$ such that for all $x \in B^{3}$, for all $r$ such that $0<r<\operatorname{dist}\left(x, \partial B^{3}\right)$, for all $r^{\prime}$ with $0<r^{\prime}<r$ we have

$$
\tilde{E}\left(x, r^{\prime}\right) \leq \tilde{E}(x, r)+\Gamma \sqrt{r} .
$$


Proof : we prove the proposition with $x=0$ and we denote $B_{r}=B(0, r), S_{r}=\partial B_{r}$ and $\tilde{E}(r)=\tilde{E}(0, r)$.

We set $\xi_{h}(x)=x \varphi_{h}(|x|)$ where

$$
\varphi_{h}=\left\{\begin{array}{l}
1 \text { on }[0, r] \\
0 \text { on }[r+h,+\infty) \\
\text { is affine on }[r, r+h]
\end{array}\right.
$$

We shall use this vector field $\xi$ as a test function in the equation verified by $u$ and we will compute the limit when $h$ tends to zero of the expression we obtain. We remark that if $g \in L^{1}\left(B^{3}\right)$,

$$
\begin{gathered}
\int_{B^{3}} g \xi_{h} \underset{h \rightarrow 0}{\longrightarrow} \int_{B(0, r)} x g \\
\int_{B^{3}} g \xi_{h_{x_{i}}}^{j} \underset{h \rightarrow 0}{\longrightarrow} \delta_{i, j} \int_{B(0, r)} g-\frac{1}{r} \int_{\partial B(0, r)} y_{i} y_{j} g d \sigma \\
\int_{B^{3}} g \operatorname{div} \xi_{h} \underset{h \rightarrow 0}{\longrightarrow} 3 \int_{B(0, r)} g-r \int_{\partial B(0, r)} g d \sigma .
\end{gathered}
$$

Hence we get the formula :

$$
\begin{gathered}
-\frac{3}{2} \int_{B_{r}}|\nabla u|^{2}+\frac{r}{2} \int_{S_{r}}|\nabla u|^{2} d \sigma+\int_{B_{r}}|\nabla u|^{2}-\frac{1}{r} \int_{S_{r}}|\nabla u \cdot y|^{2} d \sigma-3 \int_{B_{r}} \Phi \operatorname{div} u \\
\quad+r \int_{S_{r}} \Phi \operatorname{div} u+\int_{B_{r}} \Phi \operatorname{div} u-\frac{1}{r} \int_{S_{r}} \Phi<\nabla u \cdot y, y>d \sigma(y) \\
+\int_{B_{r}} x L(x) \operatorname{div} u(x) d x+\int_{B_{r}} f(u(x)) \cdot(\nabla u(x) \cdot x) d x=0 .
\end{gathered}
$$

We divide the previous formula by $r^{2}$, we use that $|\operatorname{div} u| \leq 3|\nabla u|$ and we obtain

$$
\begin{aligned}
0 \leq & \frac{d}{d r}(\tilde{E}(r))+12\|\Phi\|_{L^{\infty}} \frac{1}{r^{2}} \int_{B_{r}}|\nabla u|+\frac{6}{r} \int_{B_{r}}|L||\nabla u| \\
& +4\|\Phi\|_{L^{\infty}} \frac{1}{r} \int_{S^{r}}|\nabla u| d \sigma+\frac{1}{r} \int_{B_{r}}|f(u(x))||\nabla u| .
\end{aligned}
$$

We set now

$$
V(r)=\frac{1}{r} \int_{B_{r}}|\nabla u|
$$

We have

$$
V^{\prime}(r)=\frac{1}{r} \int_{S^{r}}|\nabla u| d \sigma-\frac{1}{r^{2}} \int_{B_{r}}|\nabla u| .
$$


Therefore the previous inequality implies that

$$
\begin{gathered}
0 \leq \frac{d}{d r}\left(\tilde{E}(r)+4\|\Phi\|_{L^{\infty}} V(r)\right) \\
+16\|\Phi\|_{L^{\infty}} \frac{1}{r^{2}} \int_{B_{r}}|\nabla u|+\frac{6}{r} \int_{B_{r}}|L||\nabla u|+\frac{1}{r}\|f\|_{\infty} \int_{B_{r}}|\nabla u| .
\end{gathered}
$$

We know that $L \in L^{3}\left(B^{3}\right)$ so $|L \| \nabla u| \in L^{\frac{6}{5}}\left(B^{3}\right)$. Hence with the Hölder inequality we obtain that there exists a constant $K$ such that

$$
0 \leq \frac{d}{d r}\left(\tilde{E}(r)+4\|\Phi\|_{L^{\infty}} V(r)+K \sqrt{r}\right) .
$$

Therefore the map

$$
r \mapsto \tilde{E}(r)+4\|\Phi\|_{L^{\infty}} V(r)+K \sqrt{r}
$$

is a non decreasing map.

Now we have

$$
V(r) \leq \frac{C}{r}\|\nabla u\|_{L^{2}\left(B^{3}\right)} \cdot r^{\frac{3}{2}}
$$

i.e. there exists a constant $\Gamma$ such that

$$
4\|\Phi\|_{L^{\infty}} V(r)+K \sqrt{r} \leq \Gamma \sqrt{r} .
$$

Therefore for all $r^{\prime}$ such that $0<r^{\prime}<r$

$$
\tilde{E}\left(r^{\prime}\right) \leq \tilde{E}(r)+\Gamma \sqrt{r}
$$

REMARK 7 : the map $r \mapsto \tilde{E}(r)+4\|\Phi\|_{L^{\infty}} V(r)+K \sqrt{r}$ is non decreasing hence it has a limit when $r$ tends to zero. Moreover the map $r \mapsto 4\|\Phi\|_{L^{\infty}} V(r)+K \sqrt{r}$ tends to zero when $r$ tends to zero, so $\tilde{E}(r)$ has a finite limit when $r$ tends to zero.

REMARK 8 : the constant $\Gamma$ does not depend on the point $x$.

\section{3 $\varepsilon$-regularity theorem.}

We will prove the following

THEOREM 4. There exist $\varepsilon_{1}, \eta, \theta$ such that for all $x \in B^{3}$ and for all $r>0$ then

$$
\left(\tilde{E}(x, r)<\varepsilon_{1}\right) \Longrightarrow(\tilde{E}(x, \theta r) \leq \sqrt{\theta} \max \{\tilde{E}(x, r), \eta \sqrt{r}\}) .
$$

Proof : we follow in this proof the ideas of L.C. Evans (see [10]).

Assume that the theorem is wrong i.e that there exist a sequence $\left(x_{k}\right)_{k \in \mathbb{N}}$ of points of $B^{3}$ and a sequence $\left(r_{k}\right)_{k \in \mathbb{N}} \in(0,1)^{\mathbb{N}}$ such that 


$$
\left\{\begin{array}{l}
\lim _{k \rightarrow+\infty} r_{k}=0 \\
\tilde{E}\left(x_{k}, r_{k}\right) \rightarrow 0 \\
\tilde{E}\left(x_{k}, \theta r_{k}\right) \geq \sqrt{\theta} \tilde{E}\left(x_{k}, r_{k}\right) \\
\frac{\tilde{E}\left(x_{k}, \theta r_{k}\right)}{\sqrt{r_{k}}} \rightarrow+\infty
\end{array}\right.
$$

We denote

$$
\lambda_{k}^{2}=\tilde{E}\left(x_{k}, r_{k}\right) \text { and } W=H-f(u) .
$$

First step : scaling method. We set

$$
v_{k}=\frac{u\left(x_{k}+r_{k} z\right)-a_{k}}{\lambda_{k}}
$$

where $a_{k}$ is the mean value of $u$ on $B\left(x_{k}, r_{k}\right)$. We remark that

$$
\forall k, \int_{B^{3}}\left|\nabla v_{k}\right|^{2}=1 \text { and } \int_{B^{3}} v_{k}=0
$$

hence $\left(v_{k}\right)_{k \in \mathbb{N}}$ is bounded in $H^{1} / \mathbb{R}$ and we may choose a subsequence such that

$$
v_{k} \rightarrow v \text { in } H^{1} \text { weak, } L^{2} \text { strong and almost everywhere. }
$$

Second step : $v$ is real harmonic in $B^{3}$.

Let $w$ be an element of $\mathcal{D}\left(B^{3}\right)$. We set $w_{k}(y)=w\left(\frac{y-x_{k}}{r_{k}}\right)$, we multiply by $w_{k}$ the equation verified by $u$ and we obtain :

$$
\begin{gathered}
\int_{B^{3}} \nabla v_{k} \cdot \nabla w-\int_{B^{3}}\left(\lambda_{k} v_{k}+a_{k}\right) \lambda_{k}\left|\nabla v_{k}\right|^{2} \cdot w \\
+\frac{r_{k}^{2}}{\lambda_{k}} \int_{B^{3}}(-W+u<u, W>)\left(x_{k}+r_{k} z\right) w(z) d z=0 .
\end{gathered}
$$

* The first term tends to

$$
\int_{B^{3}} \nabla v \cdot \nabla w
$$

* We know that

$$
\left\{\begin{array}{l}
\lim _{k \rightarrow+\infty} \lambda_{k}=0 \\
w \in \mathcal{D}\left(B^{3}\right) \text { so it is uniformly bounded in } B^{3} \\
a_{k} \text { is bounded by } 1 \\
\left|\nabla v_{k}\right|^{2} \text { is bounded in } L^{1}\left(B^{3}\right)
\end{array}\right.
$$


Therefore the second term tends to zero when $k$ tends to $+\infty$.

* Lastly the third term tends to zero also :

REMARK 9 : in this step the assumption $\frac{\tilde{E}\left(x_{k}, \theta r_{k}\right)}{\sqrt{r_{k}}} \rightarrow+\infty$ is crucial.

$$
\left|\frac{r_{k}^{2}}{\lambda_{k}} \int_{B^{3}}(-W+u<u, W>)\left(x_{k}+r_{k} z\right) w(z) d z\right| \leq 2\|w\|_{\infty} \frac{\sqrt{r_{k}}}{\lambda_{k}}\left(\int_{B_{\left(x_{k}, r_{k}\right)}}|W|^{2}\right)^{\frac{1}{2}},
$$

furthermore

$$
\tilde{E}\left(x_{k}, \theta r_{k}\right)=\frac{1}{\theta r_{k}} \int_{B\left(x_{k}, \theta r_{k}\right)}|\nabla u|^{2} \leq \frac{1}{\theta r_{k}} \int_{B\left(x_{k}, r_{k}\right)}|\nabla u|^{2}=\frac{\lambda_{k}^{2}}{\theta}
$$

and since $\frac{\tilde{E}\left(x_{k}, \theta r_{k}\right)}{\sqrt{r_{k}}} \rightarrow+\infty$ we obtain that $\frac{\lambda_{k}^{2}}{\sqrt{r_{k}}} \rightarrow+\infty$. So

$$
\frac{\sqrt{r_{k}}}{\lambda_{k}}\left(\int_{B\left(x_{k}, r_{k}\right)}|W|^{2}\right)^{\frac{1}{2}} \text { tends to zero. }
$$

Hence we see that for all $w$ in $\mathcal{D}\left(B^{3}\right)$

$$
\int_{B^{3}} \nabla v \cdot \nabla w=0
$$

Therefore $v$ is a real harmonic map on $B^{3}$.

Third step : $v_{k}$ tends strongly to $v$ in $H^{1}$.

We shall prove this result in the next subsection.

Fourth step : end of the proof. For $\nu \in(0,1)$, for $w$ in $H^{1}\left(B^{3}, \mathbb{R}^{3}\right)$, we denote

$$
E_{\nu}(w)=\frac{1}{\nu} \int_{B(0, \nu)}|\nabla w|^{2}
$$

We remark that $\tilde{E}\left(x_{k}, \theta r_{k}\right)=E_{\theta}\left(v_{k}\right)$. From the third step we obtain that $\tilde{E}\left(x_{k}, \theta r_{k}\right)$ ( resp. $\left.\tilde{E}\left(x_{k}, r_{k}\right)\right)$ tends to $E_{\theta}(v)\left(\operatorname{resp} E_{1}(v)\right)$.

Now $v$ is a real harmonic map. So there exists an universal constant $C$ such that

$$
\forall \tau \in\left(0, \frac{1}{2}\right), \frac{1}{\tau} \int_{B(0, \tau)}|\nabla v|^{2} \leq C \tau^{2} .
$$

We remark that $C$ can be chosen independent from the sequence $\left(x_{k}, r_{k}\right)_{k \in \mathbb{N}}$ because $v$ is a real harmonic map, its mean value is zero and $\|\nabla v\|_{L^{2}\left(B^{3}\right)}=1$.

With the conditions satisfied by the sequence $\left(x_{k}, r_{k}\right)_{k}$ and from the result of the third step we obtain that

$$
E_{\theta}(v) \geq \sqrt{\theta} E_{1}(v) .
$$

If we choose an a priori $\theta$ such that $C \theta^{2}<\sqrt{\theta}$ we obtain a contradiction. 
Hence Theorem 4 is proved.

We are now able to prove the following result :

THEOREM 5. ( $\varepsilon$-regularity) There exists $\varepsilon_{0}$, there exists $r_{0}>0$ such that

$$
\left(\exists r, 0<r<r_{0}, \tilde{E}(x, r) \leq \varepsilon_{0}\right) \Longrightarrow\left(\exists \Lambda_{r}, \forall s<r, \tilde{E}(x, s) \leq \Lambda_{r} \sqrt{s}\right) .
$$

The previous $\Lambda_{r}$ depends continuously on $r$ in $(0,1)$.

Proof : we set $\varepsilon_{0}=\frac{\varepsilon_{1}}{2}$ and we fix $r_{0}>0$ such that $\Gamma \sqrt{r_{0}} \leq \frac{\varepsilon_{1}}{2}$. Let $x$ in $B^{3}$ and assume that there exists $r, 0<r<r_{0}$ such that $\tilde{E}(x, r) \leq \varepsilon_{0}$. From the monotonicity formula we note that for all $s<r$ we have $\tilde{E}(x, s) \leq \varepsilon_{1}$ and we can use theorem 4 : we obtain that

$$
\forall n \in \mathbb{I}, \tilde{E}\left(x, \theta^{n} r\right) \leq \sqrt{\theta^{n}} \max \{\tilde{E}(x, r), \eta \sqrt{r}\} .
$$

Let $s \in(0, r)$ and let $n$ such that $\theta^{n} r \leq s<\theta^{n-1} r$. From the monotonicity formula we deduce that

$$
\tilde{E}(x, s) \leq \tilde{E}\left(x, \theta^{n-1} r\right)+\Gamma \sqrt{r \theta^{n-1}}
$$

i.e.

$$
\tilde{E}(x, s) \leq \frac{1}{\sqrt{\theta}} \sqrt{\theta^{n}}(\Gamma \sqrt{r}+\max \{\tilde{E}(x, r), \eta \sqrt{r}\})
$$

hence

$$
\tilde{E}(x, s) \leq \sqrt{s}\left(\Gamma+\eta+\frac{\varepsilon_{0}}{\sqrt{r}}\right) .
$$

We set $\Lambda_{r}=\Gamma+\eta+\frac{\varepsilon_{0}}{\sqrt{r}}$ and the theorem is proved.

\subsection{Compactness result.}

We prove now the third step of the previous part. This result of compactness is based on the duality $\mathcal{H}^{1}-B M O$. For more details about these spaces one can see [11]. We know for example that

THEOREM 6. If $f \in L^{\infty}\left(\mathbb{R}^{N}\right), g \in \mathcal{H}^{1}\left(\mathbb{R}^{N}\right)$,

$$
\left|\int_{\mathbb{R}^{N}} f g\right| \leq C_{2}\|f\|_{\star}\|g\|_{\mathcal{H}^{1}\left(\mathbb{R}^{N}\right)}
$$

where

$$
\|f\|_{\star}=\sup _{x \in \mathbb{R}^{N}, r>0}\left\{f_{B(x, r)}\left|f-f_{x, r}\right|\right\} .
$$

Let $\xi \in \mathcal{D}\left(R^{3}\right)$ which satisfies

$$
\left\{\begin{array}{l}
0 \leq \xi \leq 1 \\
\xi=1 \text { on } B\left(0, \frac{1}{2}\right) \\
\xi=0 \text { out of } B\left(0, \frac{5}{8}\right)
\end{array}\right.
$$


In 3.4.3 we shall multiply by $\xi^{2}\left(v_{k}-v\right)$ the Euler equation satisfied by $v_{k}-v$ in order to show that $\left\|\nabla\left(v_{k}-v\right)\right\|_{L^{2}}$ tends to zero. The most important difficulty will arise from the term

$$
=\lambda_{k} \int_{B\left(0, \frac{1}{2}\right)} \xi^{2}\left|\nabla v_{k}\right|^{2}\left(a_{k}+\lambda_{k} v_{k}\right)\left(v_{k}-v\right) .
$$

We will show that $\xi^{2}\left|\nabla v_{k}\right|^{2}\left(a_{k}+\lambda_{k} v_{k}\right)\left(v_{k}-v\right)$ is bounded in $L^{1}$ writing this term as a product of a $B M O$ function (see section 3.4.1.) by a $\mathcal{H}^{\infty}$ function (see section 3.4.2.), and using the $\mathcal{H}^{\infty}-\mathcal{B M O}$ duality.

\subsection{1 $\left(\xi v_{k}\right)_{k \in \mathbb{N}}$ is bounded in $B M O\left(\mathbb{R}^{3}, \mathbb{R}^{3}\right)$.}

Let $z_{0} \in B\left(0, \frac{7}{8}\right)$ and let $r \in\left(0, \frac{1}{8}\right)$. We set $y_{k}=x_{k}+r_{k} z_{0} \in B\left(x_{k}, \frac{7}{8} r_{k}\right)$. We remark that

$$
\frac{1}{r} \int_{B\left(z_{0}, r\right)}\left|\nabla v_{k}\right|^{2}=\frac{1}{\lambda_{k}^{2}} \frac{1}{r r_{k}} \int_{B\left(y_{k}, r r_{k}\right)}|\nabla u|^{2}
$$

By the monotonicity formula :

$$
\begin{gathered}
\frac{1}{r r_{k}} \int_{B\left(y_{k}, r r_{k}\right)}|\nabla u|^{2} \leq \frac{1}{\frac{r_{k}}{8}} \int_{B\left(y_{k}, \frac{r_{k}}{8}\right)}|\nabla u|^{2}+\left(\frac{r_{k}}{8}\right)^{\frac{1}{2}} \Gamma \\
\leq \frac{8}{r_{k}} \int_{B\left(x_{k}, r_{k}\right)}|\nabla u|^{2}+\left(\frac{r_{k}}{8}\right)^{\frac{1}{2}} \Gamma \\
\leq 8 \lambda_{k}^{2}+\left(\frac{r_{k}}{8}\right)^{\frac{1}{2}} \Gamma .
\end{gathered}
$$

Hence

$$
\frac{1}{r} \int_{B\left(z_{0}, r\right)}\left|\nabla v_{k}\right|^{2} \leq 8+\Gamma \frac{\sqrt{r_{k}}}{8 \lambda_{k}^{2}}
$$

Now $\frac{\sqrt{r_{k}}}{\lambda_{k}^{2}}$ tends to zero when $k$ tends to $+\infty$ so there exists a constant $C_{3}$ such that

$$
\forall k, \forall r \in\left(0, \frac{1}{8}\right), \forall z_{0} \in B\left(0, \frac{7}{8}\right), \frac{1}{r} \int_{B\left(z_{0}, r\right)}\left|\nabla v_{k}\right|^{2} \leq C_{3}
$$

Therefore with an inequality of Poincaré there exists a constant $C_{4}$ such that

$$
\text { (i) } \forall k, \forall r \in\left(0, \frac{1}{8}\right), \forall z_{0} \in B\left(0, \frac{7}{8}\right), f_{B\left(z_{0}, r\right)}\left|v_{k}-\left(v_{k}\right)_{z_{0}, r}\right| d z \leq C_{4}<+\infty
$$

where $\left(v_{k}\right)_{z_{0}, r}$ is the mean value of $v_{k}$ on $B\left(z_{0}, r\right)$.

Using John-Nirenberg inequality we see that $\left(v_{k}\right)_{k \in \mathbb{N}}$ is bounded in $L^{p}\left(B\left(0, \frac{7}{8}\right), \mathbb{R}^{3}\right)$ for all $p$ in $[1,+\infty)$.

Now let $z_{0} \in B\left(0, \frac{3}{4}\right)$ and $r \in\left(0, \frac{1}{8}\right]$. 
We have

$$
\begin{gathered}
f_{B\left(z_{0}, r\right)} \mid \xi v_{k}- \\
\quad\left(\xi v_{k}\right)_{z_{0}, r}\left|d z \leq f_{B\left(z_{0}, r\right)}\right| \xi v_{k}-\xi\left(v_{k}\right)_{z_{0}, r}\left|+f_{B\left(z_{0}, r\right)}\right| \xi\left(v_{k}\right)_{z_{0}, r}-\left(\xi v_{k}\right)_{z_{0}, r} \mid \\
\leq f_{B\left(z_{0}, r\right)}\left|v_{k}-\left(v_{k}\right)_{z_{0}, r}\right|+f_{B\left(z_{0}, r\right)}\left|\xi\left(v_{k}\right)_{z_{0}, r}-\left(\xi v_{k}\right)_{z_{0}, r}\right|
\end{gathered}
$$

* The first term is bounded by $C_{4}$ from $(i)$.

* Let us study the second term : we know that $\xi \in \mathcal{D}\left(\mathbb{R}^{3}\right)$ hence

$$
\begin{aligned}
\left|\xi\left(v_{k}\right)_{z_{0}, r}-\left(\xi v_{k}\right)_{z_{0}, r}\right| & (t) \leq \frac{1}{r^{3}} \int_{B\left(z_{0}, r\right)}|\xi(t)-\xi(s)| v_{k}(s) \mid d s \\
\leq & \operatorname{Lr} f_{B\left(z_{0}, r\right)}\left|v_{k}\right|
\end{aligned}
$$

since $\xi$ is a Lipschitz map and where $L$ is the Lipschitz-coefficient of $\xi$.

Therefore

$$
f_{B\left(z_{0}, r\right)}\left|\xi v_{k}-\left(\xi v_{k}\right)_{z_{0}, r}\right| d z \leq C_{4}+\frac{L}{r^{2}} \int_{B\left(z_{0}, r\right)}\left|v_{k}\right|
$$

We have already seen that $\left(v_{k}\right)_{k \in \mathbb{N}}$ is bounded in $L^{p}\left(B\left(0, \frac{7}{8}\right), \mathbb{R}^{3}\right)$ for all $p$ in $[1,+\infty)$ and in particular in $L^{3}\left(B\left(0, \frac{7}{8}\right), \mathbb{R}^{3}\right)$ by a constant $C_{5}$. Hence

$$
\begin{aligned}
\int_{B\left(z_{0}, r\right)}\left|v_{k}\right| & \leq\left(\int_{B\left(z_{0}, r\right)}\left|v_{k}\right|^{3}\right)^{\frac{1}{3}} \cdot\left(\omega_{3} r^{3}\right)^{\frac{2}{3}} \\
& \leq C_{5}\left(\omega_{3}\right)^{\frac{2}{3}} r^{2}
\end{aligned}
$$

( $\omega_{3}$ is the volume of the unit ball of $\mathbb{R}^{3}$ ).

So there exists a constant $C_{6}$ such that

$$
\forall k \in \mathbb{N}, \forall r \in\left(0, \frac{1}{8}\right), \forall z_{0} \in B\left(0, \frac{3}{4}\right), f_{B\left(z_{0}, r\right)}\left|\xi v_{k}-\left(\xi v_{k}\right)_{z_{0}, r}\right| d z \leq C_{6} .
$$

Now since $\xi=0$ out of $B\left(0, \frac{5}{8}\right)$, the same inequality is true for all $z_{0} \in \mathbb{R}^{3}$ and for all $r \in\left(0, \frac{1}{8}\right]$.

Therefore $\left(\xi v_{k}\right)_{k \in \mathbb{N}}$ is bounded in $B M O\left(\mathbb{R}^{3}, \mathbb{R}^{3}\right)$.

\subsection{2 $\mathcal{H}^{1}$ boundness.}

We recall a result of Coifman, Lions, Meyer and Semmes (see [9] and [10]).

PROPOSITION 2. Assume $u \in H^{1}\left(\mathbb{R}^{N}\right), v \in L^{2}\left(\mathbb{R}^{N}, \mathbb{R}^{N}\right)$ and div $v=0$ in $\mathcal{D}^{\prime}$. Then $\nabla u . v \in \mathcal{H}^{1}$ and

$$
\|\nabla u . v\|_{\mathcal{H}^{1}\left(\mathbb{R}^{N}\right)} \leq C_{7}\left(\|u\|_{H^{1}}^{2}\left(\mathbb{R}^{N}\right)+\|v\|_{L^{2}\left(\mathbb{R}^{N}, \mathbb{R}^{N}\right)}^{2}\right) .
$$


We set

$$
b_{k, l}^{i j}=v_{k, x_{l}}^{j}\left(a_{k}^{i}+\lambda_{k} v_{k}^{i}\right)-v_{k, x_{l}}^{i}\left(a_{k}^{j}+\lambda_{k} v_{k}^{j}\right) .
$$

First step : let us compute $\operatorname{div} b_{k, l}^{i j}$.

Let $\phi \in \mathcal{D}\left(B^{3}\right)$.

$$
\begin{gathered}
\int_{B^{3}} \phi_{x_{l}} b_{k, l}^{i j}=-\int_{B^{3}} \phi\left[v_{k, x_{l} x_{l}}^{j}\left(a_{k}^{i}+\lambda_{k} v_{k}^{i}\right)-v_{k, x_{l} x_{l}}^{i}\left(a_{k}^{j}+\lambda_{k} v_{k}^{j}\right)\right] \\
=-\int_{B^{3}} \phi\left[\triangle v_{k}^{j}\left(a_{k}^{i}+\lambda_{k} v_{k}^{i}\right)-\triangle v_{k}^{i}\left(a_{k}^{j}+\lambda_{k} v_{k}^{j}\right)\right]
\end{gathered}
$$

Now

$$
\triangle v_{k}^{j}=-\lambda_{k}\left\|\nabla v_{k}\right\|^{2}\left(a_{k}^{j}+\lambda_{k} v_{k}^{j}\right)+\frac{r_{k}^{2}}{\lambda_{k}}\left(u^{j}<u, W>-W^{j}\right)\left(x_{k}+r_{k} z\right) .
$$

Hence if we denote $\operatorname{div} b_{k, l}^{i j}=\gamma_{k}^{i j}$, we obtain

$$
\gamma_{k}^{i j}=-\frac{r_{k}^{2}}{\lambda_{k}}\left[\left(W^{j}-u^{j}<u, W>\right) u_{k}^{i}-\left(W^{i}-u^{i}<u, W>\right) u_{k}^{j}\right]_{x_{k}+r_{k} z},
$$

(we recall that $W=H-f(u)$ ).

Second step : estimation of $\left\|\gamma_{k}^{i j}\right\|_{L^{2}}$.

$$
\begin{aligned}
\left\|\gamma_{k}^{i j}\right\|_{L^{2}}^{2} & \leq 16\left(\frac{r_{k}^{2}}{\lambda_{k}}\right)^{2} \int_{B^{3}}\left|W\left(x_{k}+r_{k} z\right)\right|^{2} d z \\
& \leq 16 \frac{r_{k}}{\lambda_{k}^{2}} \int_{B\left(x_{k}, r_{k}\right)}|W(y)|^{2} d y
\end{aligned}
$$

and since $\frac{\sqrt{r_{k}}}{\lambda_{k}^{2}}$ tends to zero when $k$ tends to $+\infty$ we obtain that $\left\|\gamma_{k}^{i j}\right\|_{L^{2}}$ is bounded.

Third step : Let $\varphi_{k}^{i j} \in H_{0}^{1}\left(B^{3}\right)$ be the solution of

$$
\triangle \varphi_{k}^{i j}=\gamma_{k}^{i j}
$$

We know by standard elliptic estimates that there exists a constant $C_{8}$ such that

$$
\forall k \in \mathbb{I N}, \forall(i, j) \in\{1,2,3\}^{2},\left\|\varphi_{k}^{i j}\right\|_{H^{2}\left(B^{1}\right)} \leq C_{8} .
$$

We set $\psi_{k, l}^{i j}=\frac{\partial \varphi_{k}^{i j}}{\partial x_{l}}$ and we have

$$
\left\|\psi_{k, l}^{i j}\right\|_{H^{1}\left(B^{3}\right)} \leq C_{8} \text { and } \operatorname{div}\left(\psi_{k, l}^{i j}\right)=\gamma_{k}^{i j}
$$

We write $\beta_{k, l}^{i j}=b_{k, l}^{i j}-\psi_{k, l}^{i j}$. We have

$$
\operatorname{div}\left(\beta_{k, l}^{i j}\right)=0 \text { and } \beta_{k, l}^{i j} \in L^{2}\left(B^{3}\right) .
$$

Fourth step : now with the proposition of Coifman, Lions, Meyer and Semmes, we obtain that $\left(\xi v_{k}^{j}\right)_{x_{l}} \beta_{k, l}^{i j}$ is bounded in $\mathcal{H}^{1}$. 


\subsubsection{Compactness.}

We are going to prove that $\left(\nabla v_{k}\right)_{k \in \mathbb{N}}$ is relatively compact in $L^{2}\left(B\left(0, \frac{1}{2}\right)\right)$ for the strong topology. Let $w$ be in $H_{0}^{1}\left(B\left(0, \frac{1}{2}\right)\right)$. We multiply by $w$ the equation satisfied by $v_{k}$ and since $v$ is real harmonic we obtain

$$
\begin{gathered}
\int_{B\left(0, \frac{1}{2}\right)}\left(\nabla v_{k}-\nabla v\right) \nabla w-\lambda_{k} \int_{B\left(0, \frac{1}{2}\right)}\left|\nabla v_{k}\right|^{2}\left(a_{k}+\lambda_{k} v_{k}\right) w(z) d z \\
+\frac{r_{k}^{2}}{\lambda_{k}} \int_{B\left(0, \frac{1}{2}\right)}(-W+u<u, W>)\left(x_{k}+r_{k} z\right) w(z) d z=0 .
\end{gathered}
$$

We set $w=\xi^{2}\left(v_{k}-v\right)$ where $\xi$ is a smooth cut-off function which satisfies

$$
\left\{\begin{array}{l}
0 \leq \xi \leq 1 \\
\xi=1 \text { on } B\left(0, \frac{1}{2}\right) \\
\xi=0 \text { out of } B\left(0, \frac{5}{8}\right)
\end{array}\right.
$$

After a computation we get that the sum of four terms $I+I I+I I I+I V$ is zero.

First term :

$$
I=\int_{B\left(0, \frac{1}{2}\right)} \xi^{2}\left|\nabla v_{k}-\nabla v\right|^{2}
$$

\section{Second term :}

$$
I I=\int_{B\left(0, \frac{1}{2}\right)}\left(v_{k}-v\right) \nabla\left(\xi^{2}\right)\left(\nabla v_{k}-\nabla v\right)
$$

This term tends to zero because

$$
\left\{\begin{array}{l}
\nabla\left(\xi^{2}\right) \text { is uniformly bounded } \\
v_{k}-v \underset{k \rightarrow+\infty}{\stackrel{L^{2}}{\longrightarrow}} 0 \text { strongly } \\
\nabla v_{k}-\nabla v \underset{k \rightarrow+\infty}{\stackrel{L^{2}}{\longrightarrow}} 0 \text { weakly }
\end{array}\right.
$$

\section{Third term :}

$$
\begin{gathered}
I I I=\lambda_{k} \int_{B\left(0, \frac{1}{2}\right)} \xi^{2}\left|\nabla v_{k}\right|^{2}\left(a_{k}+\lambda_{k} v_{k}\right)\left(v_{k}-v\right) \\
=\lambda_{k} \int_{B\left(0, \frac{1}{2}\right)} \xi^{2} \sum_{i, j, l} v_{k, x_{l}}^{j} v_{k, x_{l}}^{j}\left(a_{k}^{i}+\lambda_{k} v_{k}^{i}\right)\left(v_{k}^{i}-v^{i}\right)
\end{gathered}
$$

and since $\nabla u$ is orthogonal to $u$

$$
\begin{gathered}
=\lambda_{k} \int_{B\left(0, \frac{1}{2}\right)} \xi^{2} \sum_{i, j, l} v_{k, x_{l}}^{j} b_{k, l}^{i j}\left(v_{k}^{i}-v^{i}\right) \\
=\lambda_{k}(A+B)
\end{gathered}
$$


with

$$
A=\int_{\mathbb{R}^{3}}\left(\xi v_{k}^{j}\right)_{x_{l}} b_{k, l}^{i j} \xi\left(v_{k}^{i}-v^{i}\right)
$$

and

$$
B=-\int_{\mathbb{R}^{3}} \xi_{x_{l}} v_{k}^{j} b_{k, l}^{i j}\left[\xi\left(v_{k}^{i}-v^{i}\right)\right] .
$$

$B$ is bounded by a constant independent of $k$ because

$$
\left\{\begin{array}{l}
\xi_{x_{l}} \text { is uniformly bounded } \\
\left(v_{k}^{j}\right)_{k \in \mathbb{N}} \text { is bounded in } L^{4} \\
\left(b_{k, l}^{i j}\right)_{k \in \mathbb{N}} \text { is bounded in } L^{2} \\
{\left[\xi\left(v_{k}^{i}-v^{i}\right)\right]_{k \in \mathbb{N}} \text { is bounded in } L^{4}}
\end{array}\right.
$$

Let us study $A$ :

$$
A=\int_{\mathbb{R}^{3}}\left(\xi v_{k}^{j}\right)_{x_{l}} \beta_{k, l}^{i j} \xi\left(v_{k}^{i}-v^{i}\right)+\int_{\mathbb{R}^{3}}\left(\xi v_{k}^{j}\right)_{x_{l}} \psi_{k, l}^{i j} \xi\left(v_{k}^{i}-v^{i}\right)
$$

The first term is bounded because $\left(\xi v_{k}^{j}\right)_{x_{l}} \beta_{k, l}^{i j}$ is bounded in $\mathcal{H}^{1}$ and $\xi\left(v_{k}^{i}-v^{i}\right)$ is bounded in $\mathrm{BMO}$ and these two spaces are in duality.

The second term is bounded because $\left(\xi v_{k}^{j}\right)_{x_{l}}$ is bounded in $L^{2}, \psi_{k, l}^{i j}$ is bounded in $L^{6}$ and $\xi\left(v_{k}^{i}-v^{i}\right)$ is bounded in $L^{3}$.

Now it is clear that $I I I$ tends to zero when $k$ tends to $+\infty$.

Fourth term :

$$
I V=\frac{r_{k}^{2}}{\lambda_{k}} \int_{B\left(0, \frac{1}{2}\right)}(-W+u<u, W>)\left(x_{k}+r_{k} z\right) \xi^{2}\left(v_{k}-v\right)
$$

hence

$$
|I V| \leq 2 \frac{r_{k}^{2}}{\lambda_{k}}\left\|W\left(x_{k}+r_{k} z\right)\right\|_{L^{2}\left(B\left(0, \frac{1}{2}\right)\right)} \cdot\left\|v_{k}-v\right\|_{L^{2}\left(B\left(0, \frac{1}{2}\right)\right)}
$$

So

$$
|I V| \leq 2 \frac{\sqrt{r_{k}}}{\lambda_{k}}\|W\|_{L^{2}\left(B\left(x_{k}, r_{k}\right)\right)} \cdot\left\|v_{k}-v\right\|_{L^{2}\left(B\left(0, \frac{1}{2}\right)\right)} \cdot
$$

This term tends to zero since $v_{k}$ tends to $v$ in $L^{2}$ strong and since $\frac{\sqrt{r_{k}}}{\lambda_{k}}$ tends to zero.

As a conclusion we obtain that

$$
\nabla v_{k} \underset{k \rightarrow+\infty}{\longrightarrow} \nabla v \text { in } L^{2} \text { strong }
$$

and the result is proved. 


\subsection{End of the proof of Theorem 3 .}

We set

$$
A=\left\{x, \exists r, 0<r<r_{0}, \tilde{E}(x, r)<\varepsilon_{0}\right\} .
$$

For all $r>0, x \mapsto \tilde{E}(x, r)$ is a continuous map, hence $A$ is an open set of $B^{3}$.

Now by a standard covering argument we know that $\mathcal{H}^{1}\left(B^{3} \backslash A\right)=0$ (see [14]).

Finally if $x_{1} \in A$, we fix $r_{1}<r_{0}$ such that $\tilde{E}\left(x_{1}, r_{1}\right)<\varepsilon_{0}$. Using the continuity of $x \mapsto \tilde{E}\left(x, r_{1}\right)$ we know that there exists $\mu>0$ such that for all $x \in B\left(x_{1}, \mu\right), \tilde{E}\left(x, r_{1}\right)<\varepsilon_{0}$. From the theorem of $\varepsilon$-regularity we know that for all $s<r_{1}$, for all $x \in B\left(x_{1}, \mu\right)$,

$$
\tilde{E}(x, s) \leq \Lambda_{r_{1}} \sqrt{s}
$$

and with the integral characterization of the Hölder continuous maps we obtain that $u \in$ $\mathcal{C}^{0, \frac{1}{2}}\left(B\left(x_{1}, \mu\right)\right)$.

Therefore if $x \in A$ then $u$ is Hölder continuous in a neighbourhood of $x$ and theorem 3 is proved.

\section{Further results about the minimizers of $E$.}

In conclusion we mention in this part any results which concern the minimizers of the micromagnetism energy.

Let $g \in H^{\frac{1}{2}}\left(\partial B^{3}, S^{2}\right)$. We write $H_{q}^{1}\left(B^{3}, S^{2}\right)$ the set of the maps $v$ in $H^{1}\left(B^{3}, S^{2}\right)$ such that $v=g$ on $\partial B^{3}$. We consider $u \in H_{g}^{1}\left(B^{3}, S^{2}\right)$. Let us assume that $u$ is a minimizer of $E$ on $H_{g}^{1}\left(B^{3}, S^{2}\right)$.

We already know that $u$ is regular on $B^{3} \backslash V$, where $\mathcal{H}^{1}(V)=0$, since $u$ is also a stationary critical point of $E$.

First we can prove that $u$ satisfies monotonicity formulae better than in the stationary case.

PROPOSITION 3. For all $\alpha$ in $(0,2)$ there exists a constant $\Gamma_{\alpha}$ such that

$$
r \mapsto \tilde{E}\left(x_{0}, r\right)+\Gamma_{\alpha} r^{\alpha}
$$

is a non decreasing function of $r$.

Sketch of the proof : we proceed like in the minimizing harmonic maps case. We fix $x$ in $B^{3}$ and $r \in(0,1-|x|)$, we set

$$
v(y)=\left\{\begin{array}{l}
u\left(x+r \frac{y-x}{|y-x|}\right) \text { if } y \in B(x, r) \\
u(y) \text { if } y \notin B(x, r)
\end{array}\right.
$$

Since $u$ is a minimizer of $E$ we write

$$
E(u) \leq E(v)
$$


We obtain then that there exists a constant $\Gamma_{\alpha}$ such that

$$
0 \leq \frac{d}{d r}\left(\tilde{E}(x, r)+\Gamma_{\alpha} r^{\alpha}\right)
$$

The term $\frac{d}{d r} \tilde{E}(x, r)$ comes from the Dirichlet energy. The perturbation $\frac{d}{d r}\left(\Gamma_{\alpha} r^{\alpha}\right)$ is due to the last two terms of $E$.

REMARK 10 : in order to treat the non local term we remark that

$$
\|u-v\|_{L^{2}\left(B^{3}\right)} \leq K r^{\frac{3}{2}} \quad \text { hence } \quad\left\|H_{u}-H_{v}\right\|_{L^{2}\left(B^{3}\right)} \leq C r^{\frac{3}{2}} .
$$

Like in the case of minimizing harmonic maps, we show that the singularities of $u$ are isolated.

THEOREM 7. Assume $u \in H^{1}\left(B^{3}, S^{2}\right)$ is a minimizer of $E$. Then the singularities of $u$ are isolated.

Sketch of the proof : assume that for example zero is a non isolated singularity of $u$. We set

$$
u_{\sigma}(x)=u(\sigma x)
$$

We remark that

$$
\int_{B(0,1)}\left|\nabla u_{\sigma}\right|^{2}=\frac{1}{\sigma} \int_{B_{\sigma}}|\nabla u|^{2}
$$

and the last term is bounded thanks to the monotonicity formula. Then we can extract a subsequence $\left(u_{\sigma_{n}}\right)_{n}$ which converges to $u_{0}$ in $H^{1}$ weak, $L^{2}$ strong and almost everywhere.

Then we prove that we can extract this subsequence such that the convergence is in $H^{1}$ strong and $u_{0}$ is a radial minimizing harmonic map.

Now the idea underlying the proof is that if there is a sequence of singularities which converges to zero, then $u_{0}$ has a line of singularities, and this is impossible (see [14]).

REMARK 11 : these results are proved at length in [8]. I recall that similar results about the minimizers of $E$ are obtained by Hardt and Kinderlehrer in [13], with a very different and very interesting approach.

Acknowledgements. The author is pleased to express his gratitude to F.Hélein for his helpful suggestions. He would like to thank also F. Bethuel, T. Horsin and T. Rivière for interesting discussions.

\section{References}

[1] F. J. Almgren, Jr. and E. H. Lieb : How many Singularities can be there in an Energy Minimizing Maps from the Ball to the Sphere? Ideas and methods in mathematical 
analysis, stochastics and applications, In memory of R. Hoeegh-Krohn, 1 (1992), 394408.

[2] S. Baraket : Estimations of the best constant involving the $L^{\infty}$ norm in Wente's inequality, to appear in Annales de la Faculté des Sciences de Toulouse.

[3] F. Bethuel : Un résultat de regularité pour les solutions de l'équation des surfaces à courbure moyenne prescrite, C. R. Acad. Sci. Paris, série I, 314 (1992), 1003-1007.

[4] F. Bethuel : On the Singular Set of Stationary Harmonic Maps, Manuscripta Math. 78 (1993), 417-443.

[5] H. Brezis, J. M. Coron : Multiple Solutions of H- Systems and Rellich's Conjecture, Comm. Pure Appli. Math., 37 (1984), 149-187.

[6] W. Fuller Brown, Jr. : Micromagnetics, interscience publishers, 1963.

[7] G. Carbou : Regularity for a non linear variational problem : Manuscripta Math. 78 (1993), 37-56.

[8] G. Carbou : Thèse de doctorat, ENS Cachan, 1995.

[9] R. Coifman, P.L. Lions, Y. Meyer et S. Semmes : Compacité par compensation et espaces de Hardy, C. R. Acad. Sci. Paris, série I, 309 (1989), 935-949.

[10] L. C. Evans : Partial Regularity for Stationary Harmonic Maps into Spheres, Arch. Rat. Mech. Anal. 116 (1991) 101-163.

[11] C. Fefferman, E. Stein : $\mathcal{H}^{p}$ spaces of several variables : Acta Math. 129 (1972), 137-193.

[12] M. Giaquinta : Multiple Integrals in the Calculus of Variations and Nonlinear Elliptic Systems, Princeton Univ. Press, 1989.

[13] R. Hardt, D. Kinderlehrer : Some Regularity Results in Ferromagnetism, to appear.

[14] R. Hardt, D. Kinderlehrer, F. H. Lin : Existence and Partial Regularity of Static Liquid Crystal Configurations, Commun. Math. Phys, 105 (1986), 547-570.

[15] F. Hélein : Régularité des applications faiblement harmoniques entre une surface et une variété riemannienne. C. R. Acad. Sci. Paris, série I. 312 (1991), 591-596.

[16] F. Hélein : Régularité des applications faiblement harmoniques entre une surface et une sphère : C. R. Acad. Sci. Paris, série I.311 (1990), 519-524.

[17] F. Hélein : Regularity of Weakly Harmonic Maps from a Surface into a Manifold with Symmetries : Manuscripta Math. 70 (1991), 203-218.

[18] O. A. Ladyzenskaya, N. N. Ural'ceva : Equations aux dérivées partielles de type elliptique. Dunod, Paris (1968). 
[19] J. Miltat : Domains and domain walls in soft magnetic materials, mostly, Proceedings of the NATO ASI : Applied Magnetism (Erice, 1992), R. Gerber,C.D. Wright et G. Asti Eds, Kluwer Press.

[20] C. B. Jr. Morrey : The problem of Plateau on a Riemannian Manifold, Ann. of Math. 49 (1948), 807-851.

C. B. Jr. Morrey : Multiple Integrals in the Calculus of Variations, Grundlehren 130 (Springer, Berlin, 1966).

[21] J. Qing : Boundary Regularity of Weakly Harmonic Maps from Surfaces, preprint.

[22] T. Rivière : Applications harmoniques de $B^{3}$ dans $S^{2}$ partout discontinues, C.R. Acad. Sci. Paris, série I, 314 (1992), 719-723.

[23] R. Schoen, K. Uhlenbeck : A regularity theory for harmonic maps, J. diff. geom. 17 (1982), 307-335.

[24] E. M. Stein : Singular integrals and differentiability properties of functions, Princeton University Press, Princeton (1970). 